\title{
Nanoscale dopant profiling of individual semiconductor wires by capac- itance-voltage measurement
}

\author{
Timothée Lassiaz ${ }^{1,2}$, Pierre Tchoulfiann ${ }^{1}$, Fabrice Donatini², Julien Brochet ${ }^{1}$, Romain Parize ${ }^{1}$, Gwénolé Jacopin ${ }^{2}$ \\ and Julien Pernot ${ }^{2}$
}

${ }^{1}$ Aledia, F-38130 Echirolles, France

2Univ. Grenoble Alpes, CNRS, Grenoble INP, Institut Néel, F-38000 Grenoble, France

\begin{abstract}
Developing nanoscale electrical characterization techniques adapted to three-dimensional (3D) geometry is essential for optimization of the epitaxial structure and doping process of nano- and microwires. In this paper, we demonstrate the assessment of the depletion width as well as the doping profile at the nanoscale of individual microwire core-shell light-emitting devices by capacitance-voltage measurements. A statistical study carried out on sin-
\end{abstract} gle wires shows the consistency of the dop-
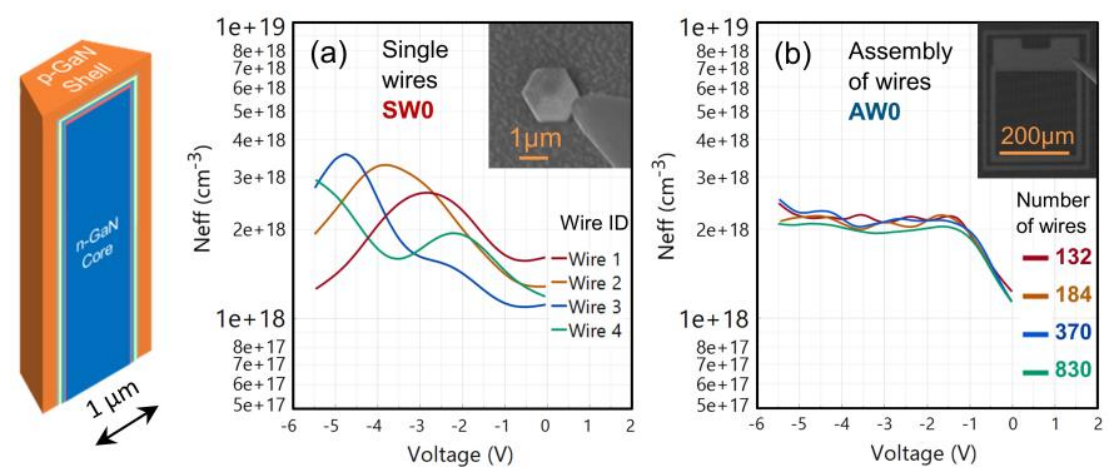

ing profile values measured for individual microwires as compared to assemblies of hundreds of wires processed on the same sample. The robustness of this method is then demonstrated on four epitaxial structures with different growth and doping conditions. Finally, electron beam-induced current and secondary electrons profiles are used to validate the depletion region width and the position in the core-shell structure.

KEYWORDS: Capacitance-voltage, Electron Beam Induced Current, Dopant profiling, LED, GaN, Core-shell wires

The promising optoelectronic properties of Gallium Nitride (GaN) and its alloys have driven research activities worldwide since the first GaN-based light emitting diodes (LEDs) demonstrated in the early 1990s by Nakamura et $\mathrm{al}^{1}$. After thirty years of development, the maturity of GaN-based LEDs for lighting applications makes these devices a potential candidate for the $\mu$ LED display technology 2,3. Despite their high efficiency ${ }^{2,4,5}$ and brightness ${ }^{4-6}$, two-dimensional (2D) c-plane $\mu$ LEDs are currently facing numerous challenges including efficiency reduction with pixel downscaling 7 and difficult pick-andplace manufacturing technologies ${ }^{8}$. Core-shell wire-based LEDs have emerged as an attractive alternative to c-plane 2D LEDs for photonic device applications and especially $\mu$ LED displays ${ }^{2,3,9}$. The high aspect ratio due to the threedimensional (3D) geometry could increase the active region area which is in turn expected to alleviate the efficiency droop issue in LEDs ${ }^{10}$. Also core-shell wire-based LEDs should not suffer from a reduction in efficiency when downscaled given that there are no surface effects in the active region.

The potential of core-shell wires devices derives from a spatially resolved nanoscale understanding of their promising properties ${ }^{11}$. Developing characterization techniques adapted to wires is thus essential to optimize their structures and improve their performances. The assessment of optical, structural, and electrical properties is crucial for the enhancement of the quality of optoelectronic devices. Characterization techniques such as cathodoluminescence ${ }^{12-14}$, time-resolved cathodoluminescence ${ }^{12,15}$, photoluminescence ${ }^{16-18}$, energy-dispersive $\mathrm{X}$-ray ${ }^{15}$, scanning near-field optical microscopy ${ }^{19}$, scanning photocurrent microscopy $^{20}$, field effect transistors ${ }^{21}$ and thermo-electric Seebeck effects ${ }^{22}$ have been successfully adapted from the planar structures to the wire-based geometry. The manufacture of 3D p-n junctions embedded in core-shell wires makes crucial the development of new techniques adapted to measure the doping level of the different epitaxial layers of the LED stack. Kelvin Probe Force Microscopy (KPFM) has been used on axial nanowires to determine the size and location of the space charge area ${ }^{23}$. However, KPFM is only applicable to the apparent p-n junction and this is not relevant for core-shell wires. Electron beam induced current (EBIC) ${ }^{11,15}$ and Hall-effect ${ }^{14,24}$ measurements have been performed on core-shell wire-based structures to respectively determine the $\mathrm{p}-\mathrm{n}$ junction position with the associated diffusion lengths and the free carrier densities but these techniques are time consuming which does not allow for an indepth statistical study. Capacitance-Voltage (C-V) measurement allows the width of the space charge region (SCR) and the dopant distribution in the structure to be extracted and 
was intensively used to characterize planar optoelectronic devices $^{25-31}$. C-V measurement appears as the most suitable technique to characterize assemblies of wires in which each single wire contributes to the signal. Even, if $\mathrm{C}-\mathrm{V}$ technique of a single thick InGaN quantum well of $8 \mathrm{~nm}$ with $10 \%$ of indium content. This was incorporated between two GaN barriers of $7 \mathrm{~nm}$ and $6 \mathrm{~nm}$ respectively followed by a $20 \mathrm{~nm}$ AlGaN electron blocking layer (EBL) with $19 \%$ of aluminium (a)

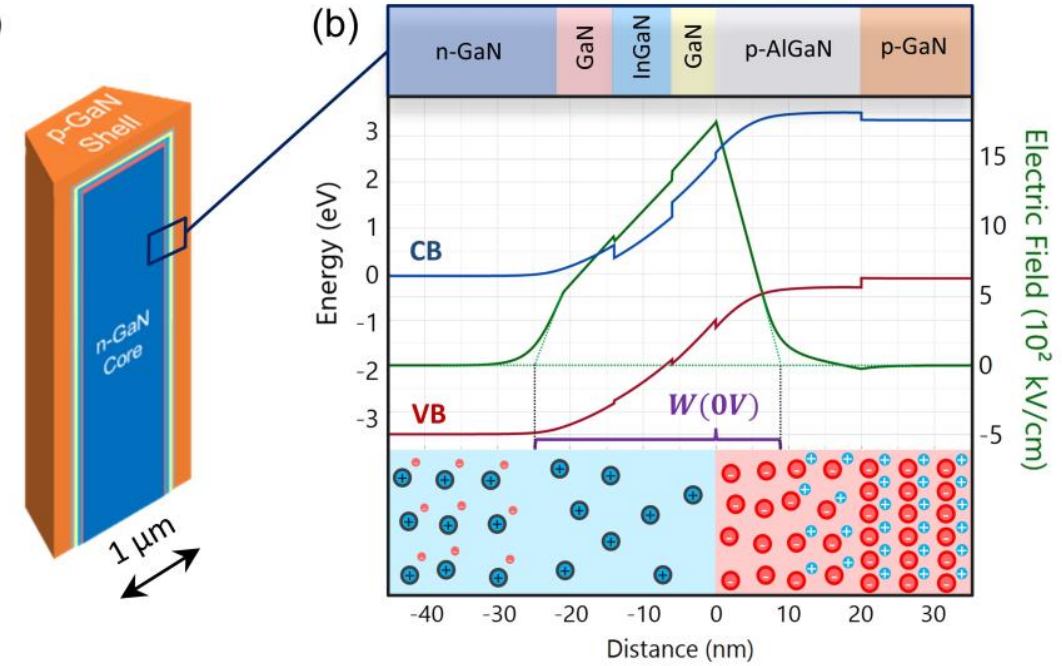

(c)

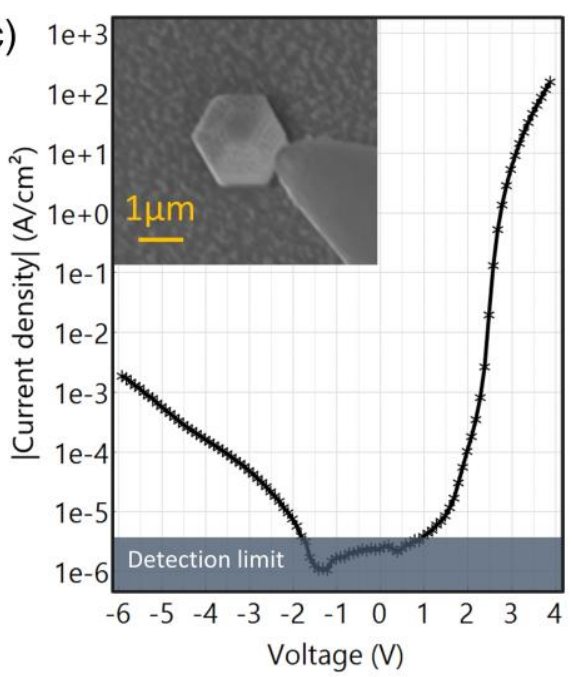

Figure 1. (a) Illustration of a 3-dimensionnal cleaved InGaN/GaN core-shell wire. (b) Schematic cross-section of the LED stack with dimensions and doping levels from sample SW1 in Table 1, the corresponding energy bands and electrical field profile at 0V. (c) Current-Voltage characteristic of a single InGaN/GaN core-shell wire and SEM picture of a single wire electrically connected. The pcontact is taken using a microtip in contact with the TCO layer deposited on the p-GaN, while the n-contact is directly taken on the silicon substrate.

has been already performed on assemblies of wires ${ }^{32}$, the bridge between the contribution to the $\mathrm{C}-\mathrm{V}$ signal of numerous single wires to assemblies of wires have never been investigated. Here, we report the assessment of dopant concentration as well as the width of the depletion region at the nanoscale on a single 3D core-shell microwire and we investigate the reliability to perform $\mathrm{C}-\mathrm{V}$ measurement on assemblies of wires with the objective of developing characterization techniques on an industrial scale.

In this paper, we studied the electrical properties of both single (SW) and assembly (AW) of InGaN/GaN coreshell wires grown by metal-organic vapor phase epitaxy (MOVPE). First, a brief description of the electrostatic properties of the nitride-based LED and its current-voltage (I-V) characteristics are presented. Then, the results of $\mathrm{C}-\mathrm{V}$ measurements on a single wire and an assembly of wires enabled us to extract the dopant concentration and the associated depletion width calculated by using the classical $\mathrm{p}$ $\mathrm{n}$ junction theory. Following the validation of this method supported by simulations, the robustness of this technique is demonstrated by comparing samples from different growth conditions. Finally, the values of the depletion width obtained by $\mathrm{C}-\mathrm{V}$ and EBIC measurements for the same sample are compared.

The InGaN/GaN core-shell wires were grown using catalyst-free MOVPE 33,34 on a [111] silicon substrate with a resistivity of $\rho=1 \mathrm{~m} \Omega . \mathrm{cm}$. A selective growth area through a mask (with sub-micrometer openings) was used to grow wurtzite $n$-doped GaN core along the [0001] direction ( $\mathrm{N}$ polar orientation) with a radius of $250 \mathrm{~nm}$ (defined as hexagon side length) and a height of $10 \mu \mathrm{m}$. Figure $1 \mathrm{a}$ shows that the active region is encapsulated between a ndoped GaN core and p-doped GaN shell. For the sake of simplicity, as shown in Figure $1 \mathrm{~b}$ the active region only consists content. This active region was then covered by a p-doped GaN shell grown over both the non-polar lateral sidewalls and the polar upper surface. The final radius of the circumscribed circle of the hexagonal base of each wire was around $900 \mathrm{~nm}$ while the height was around $10 \mu \mathrm{m}$. Additional details of the growth conditions are reported in supporting information 1.

In order to understand the electrostatic properties of the whole stack, we performed finite element calculation. Figure $1 \mathrm{~b}$ shows the energy band diagram at $0 \mathrm{~V}$ of a radial (m-plane) junction corresponding to sample SW1 from Table 1, with the associated valence band (VB) and conduction band (CB). This was obtained using Nextnano software ${ }^{35}$. The curvature of the band diagram resulting from the InGaN QW and the AlGaN EBL was characteristic of a non-polar p$n$ junction. With the extension of the tangent to the electric field toward $0 \mathrm{kV} / \mathrm{cm}$, we could infer the depletion width on both n-side and p-side. The schematic distribution of dopants in the active region is also shown in Figure $1 \mathrm{~b}$.

In order to measure the electrical properties of the p-n junction, samples have been processed (see clean room processes in supporting information 2). I-V measurements have been performed on single wires, a typical I-V curve is shown in Figure 1c and discussed quantitatively in supporting information 3 . The leakage current in reverse regime is sufficiently low to ensure a reliable $\mathrm{C}-\mathrm{V}$ measurement. $\mathrm{C}-\mathrm{V}$ is an efficient method for the assessment of doping levels and the depletion width of a p-n junction ${ }^{25}$. In semiconductor theory, the measured capacitance is the sum of the junction capacitance and the diffusion capacitance ${ }^{36}$. Only the measurements versus reverse voltage are presented in this paper. In these conditions, the contribution of the diffusion capacitance (which is dominating in the forward regime ${ }^{25}$ ) 
was assumed to be negligible. Thus, the physical mechanisms at the origin of a diffusion capacitance in forward voltage which have been detailed in previous reports ${ }^{25,37}$ are not discussed herein. If we only consider the SCR of the electric field were consequently modified (Figure 3a and $3 \mathrm{~b}$ ). From the variation of the junction capacitance with the applied reverse voltage, the apparent dopant concentration $N_{\text {eff }}$ can be analytically determined. This quantity is in-

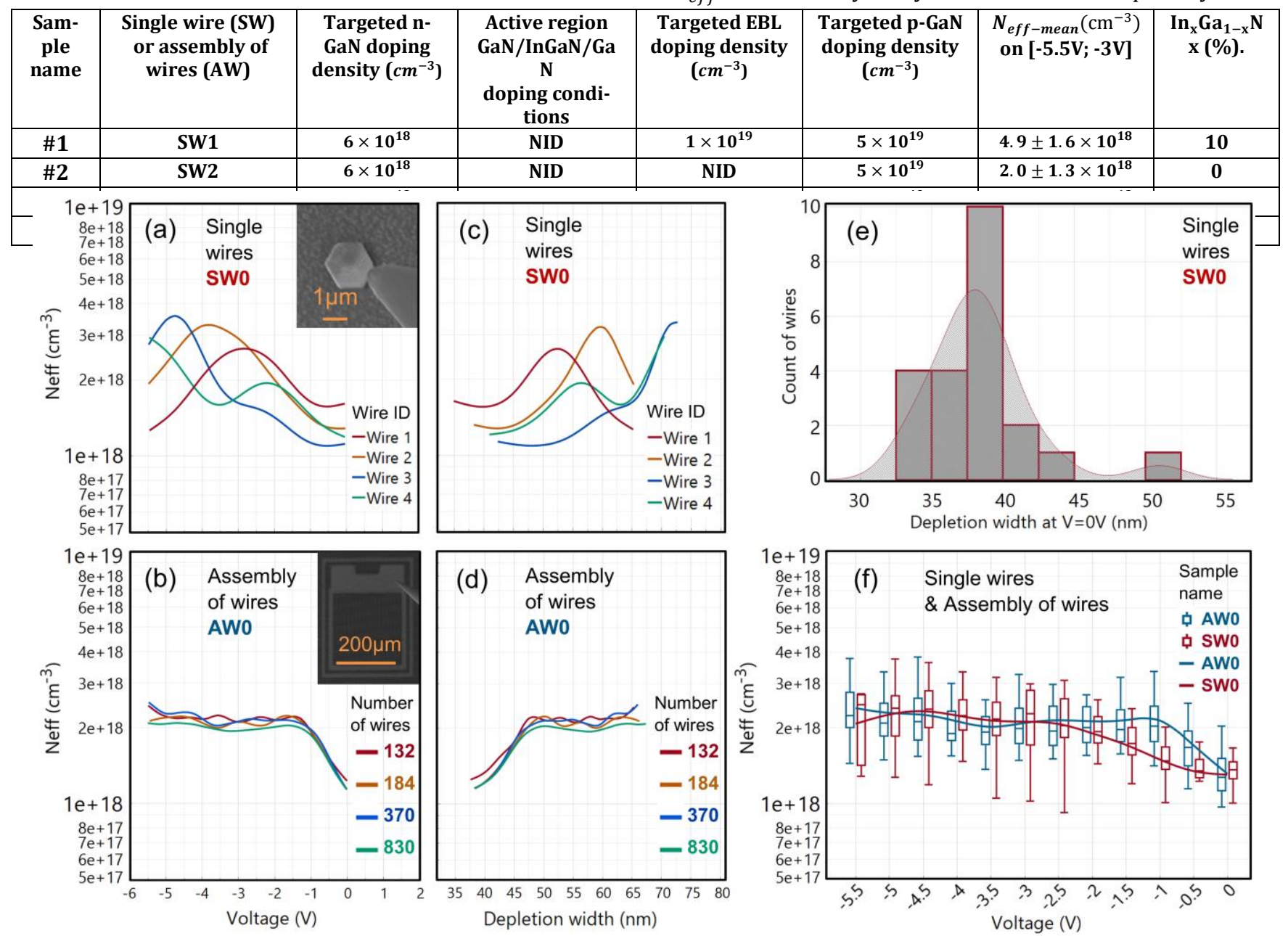

Figure 2. $N_{\text {eff }}$ vs bias of four different single wires of the validation sample SW0 (a) and four different assemblies of wires with different number of connected wires of the validation sample AW0 (b). $N_{\text {eff }}$ vs depletion width of the same four single wires (c) and the same four assemblies of wires (d). (e) Statistical distribution (histogram and kernel density estimate) of the mean values of the depletion width $W$ at $0 \mathrm{~V}$ for 22 single wires of the sample SW0. (f) Comparison of the statistical distribution of $N_{e f f}$ vs voltage between AW0 and SW0. The mean values of $N_{\text {eff }}$ is also highlighted by the smooth curves between $0 \mathrm{~V}$ and $-5.5 \mathrm{~V}$. The boxplots are standard conventions showing the $N_{\text {eff }}$ distribution at each voltage from $0 \mathrm{~V}$ to $-5.5 \mathrm{~V}$ by $0.5 \mathrm{~V}$ step. The calculation of $N_{e f f}$ is made at the same voltages for each sample. They are not superimposed for clarity.

p-n junction and not the accumulated free carriers (two-dimensional electron or hole gas), the measured capacitance can be expressed as ${ }^{28}$ :

$$
C_{j}(V)=\frac{\epsilon \epsilon_{0} S}{W}
$$

with $\epsilon_{0}$ the vacuum permittivity, $\epsilon=9.5$ the GaN relative permittivity ${ }^{28,38}, S$ the active surface of the junction, $W$ the depletion region width and $V$ the applied reverse voltage. The value of the depletion width was then directly extracted from the capacitance measurement. The SCR was enlarged as the reverse voltage increased and the band diagram and ferred from the measured $\mathrm{C}-\mathrm{V}$ curve using the formula ${ }^{26}$ :

$$
N_{e f f}(V)=-\frac{2}{|q| \epsilon \epsilon_{0} S^{2}}\left[\frac{d\left(\frac{1}{c^{2}}\right)}{d V}\right]^{-1}
$$

where $|q| \cong 1.602 e^{-19} \mathrm{C}$ was the elementary charge. The relative permittivity $\epsilon$ was assumed to be constant within the whole structure (InGaN, GaN, AlGaN). Indeed, the indium content in the QW and the aluminium content in the EBL induced a negligible impact on the values of the relative permittivity $\epsilon_{\text {GaN }} \cong \epsilon_{\text {AlGaN }} \cong \epsilon_{\text {InGaN }}$ which means $N_{\text {eff }}$ only

Table 1. Structures of samples 1 to 4 : The labels SW and AW indicate if the processing and C-V measurement was carried out on a single wire or an assembly of wires respectively. The table lists the doping density targeted from growth conditions for $\mathrm{n}$-GaN, active region GaN/InGaN/GaN, EBL and p-GaN layers, $N_{\text {eff-mean }}$ on the voltage range [-5.5V; $\left.-3 \mathrm{~V}\right]$ and the indium content in the QW. Non-intentionally doped (NID) regions can be either n-type or p-type depending on the Mg back diffusion. 
depends on the dopant concentration in the structure with $\frac{1}{N_{e f f}}=\frac{1}{N_{A}}+\frac{1}{N_{D}}$ where $N_{A}$ the acceptor concentration and $N_{D}$ the donor concentration. An accurate value of the active surface $S$ must be introduced in equations (1) and (2) to reliably evaluate the depletion width and the effective dopant concentration. The assessment of the active surface
$3 \mathrm{~d}$ report simulations that have been carried out to better understand the origin of the doping profile fluctuations.

$\mathrm{C}(\mathrm{V})=\mathrm{dQ} / \mathrm{dV}$ was simulated in a one-dimensional (1D) structure from $-5.5 \mathrm{~V}$ to $0 \mathrm{~V}$, by step of $0.1 \mathrm{~V}$ thanks to Nextnano++ finite element software. Then $N_{e f f}$ was inferred from equation (2) in order to check the impact of the EBL thickness and the p-type doping level in the EBL.
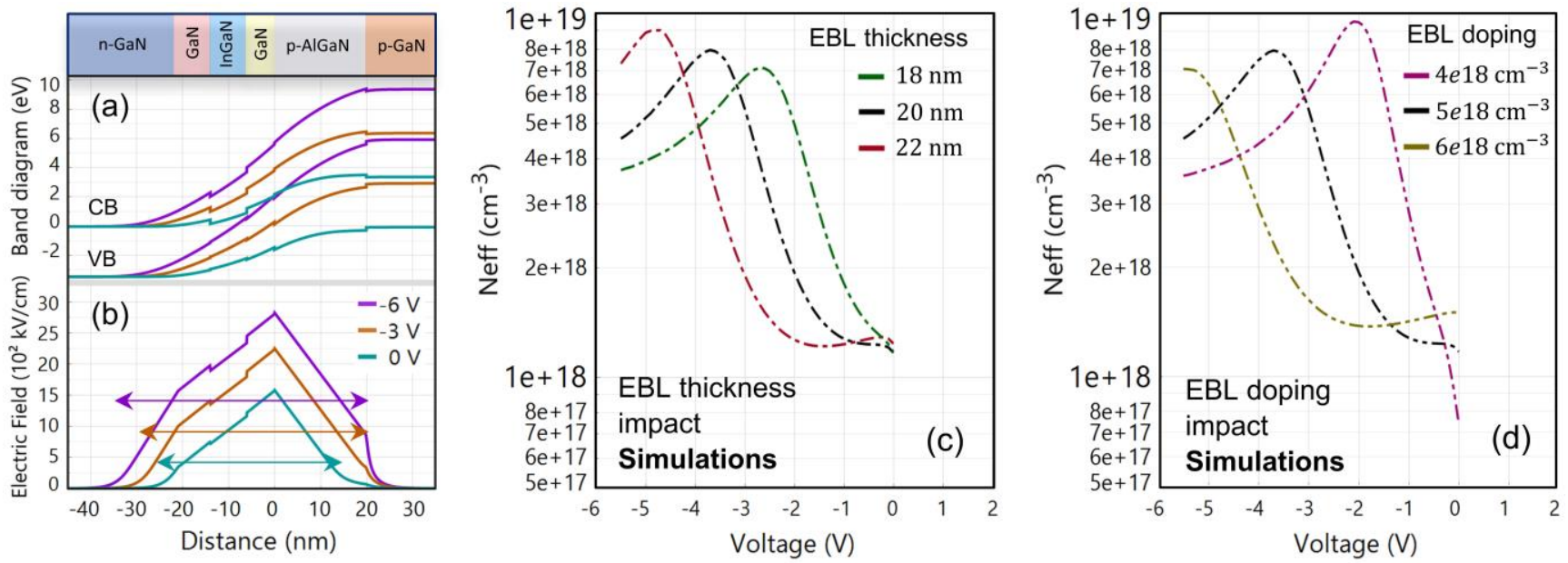

Figure 3. Simulation of (a) the band diagram and (b) the associated electric field of the structure detailed below, showing the enlargement of the SCR with the applied reverse voltage (indicated by the two-headed arrows). The doping level of n-GaN, active region and p-GaN layers are respectively $N_{D}=6 \times 10^{18} \mathrm{~cm}^{-3}, N_{D}=3 \times 10^{18} \mathrm{~cm}^{-3}$ and $N_{A}=5 \times 10^{19} \mathrm{~cm}^{-3}$. The default value of doping level and thickness of the EBL are respectively $N_{A}=5 \times 10^{18} \mathrm{~cm}^{-3}$ and $20 \mathrm{~nm}$. The active region consists in $7 \mathrm{~nm}$ GaN, 8 $\mathrm{nm}$ InGaN and $6 \mathrm{~nm} \mathrm{GaN}$, with a 10\% indium content in the QW. Simulation of $N_{\text {eff }}$ versus reverse applied voltage for 1D structures with (c) different EBL thicknesses $(18 \mathrm{~nm}, 20 \mathrm{~nm}, 22 \mathrm{~nm})$ and constant EBL doping level $\left(N_{A}=5 \times 10^{18} \mathrm{~cm}^{-3}\right)$ and (d) different EBL doping levels $\left(N_{A}=4 \times 10^{18} \mathrm{~cm}^{-3}, 5 \times 10^{18} \mathrm{~cm}^{-3}, 6 \times 10^{18} \mathrm{~cm}^{-3}\right)$ and constant thickness $(20 \mathrm{~nm})$.

of the junction was carried out from the wire's geometry and its calculation is detailed in the supporting information 4. The spatial resolution of the $\mathrm{C}-\mathrm{V}$ profiling was limited by the Debye lengths as discussed in supporting information 5.

In order to implement the $\mathrm{C}-\mathrm{V}$ measurement on core-shell wires (experimental details are given in supporting information 6), a sample \#0 has been investigated to compare the values of $N_{\text {eff }}$ at different voltages between assemblies of wires (AW0) and single wires (SW0). This validation sample aims to investigate the consistency between the results obtained for single wires and assemblies of wires coming from the same epitaxy run. The number of single wires, assembly of wires and number of connected wires per assembly are reported in supporting information 7 . The value of the depletion width was determined from equation (1). At OV the SCR is large enough to encompass the QW. This is supported by an electro-optical measurement detailed in the supporting information 8. As shown in Figure $2 \mathrm{a}$ and Figure $2 \mathrm{~b}$ respectively, the increase of $N_{\text {eff }}$ with the reverse applied voltage began between $-1 \mathrm{~V}$ and $0 \mathrm{~V}$ for the single wires and at exactly $0 \mathrm{~V}$ for the assembly of wires. In reverse voltage between $0 \mathrm{~V}$ and $-3 \mathrm{~V}$, the nanoscale resolved enlargement of the SCR seen in Figure 2c and $2 \mathrm{~d}$ allows an increase in the effective dopant concentration probed until reaching a maximum value to be determined. Each curve in Figure $2 \mathrm{a}$ and $2 \mathrm{c}$ has a similar trend but exhibits variations in the peak position, values of $N_{e f f}$ at a given voltage and depletion width on the voltage range [-5.5V; $0 \mathrm{~V}]$. Moreover, the depletion width at $0 \mathrm{~V}$ vary from one wire to another as shown in the statistical distribution Figure 2e. Figure 3c and
As shown in Figure 3c, a variation in the thickness of the EBL of a few nm leads to a significant shift of the peak position and the maximum value of $N_{e f f}$. In the same way, the p-type doping level in EBL impacts the position and maximum value of the peak, as well as the slope of the effective doping level (Figure 3d). This suggests that the slope depends more on the doping than on the thickness of the layer. These simulations highlight the sensitivity of the $\mathrm{C}-\mathrm{V}$ measurement and the strong interdependence of the structural parameters. Measurement performed on assembly of wires, having fluctuations on thicknesses and doping levels from one wire to another, must lead to an average value and hence a flattening of the peaks. Figure $2 \mathrm{~b}$ and Figure $2 \mathrm{~d}$ present four different doping profile measurement on assemblies of wires. From one device to another, the number of electrically connected wires varied from $\sim 100$ to $\sim 850$. The doping profile $N_{\text {eff }}$ has the same trend for all the devices, versus applied voltage and versus depletion width. Contrary to the single wire, the assembly of wires does not show a single peak on the voltage range $[-5.5 \mathrm{~V} ;-2 \mathrm{~V}]$ but there was a plateau within the range $[-5.5 \mathrm{~V} ;-1.5 \mathrm{~V}]$. It could result from the contribution of each connected wire having its peak position at a different voltage. The flatness of the plateau increased with the number of wires connected due to the higher average of peaks at different position. Figure $2 \mathrm{f}$ shows the mean values of $N_{\text {eff }}$ at different voltages for AW0 and SW0 of samples $\# 0$. Below an applied voltage of $-3 \mathrm{~V}$, the $N_{\text {eff-mean }}$ are very close for both devices but the boxplots show a higher disparity for the single wires. This was to be expected given that there was a larger number of connected wires in each 
assembly and that the measured capacitance is the sum of the capacitance of each electrically connected wire.

Then, in the light of the investigations described above for sample \#0, we implemented the $\mathrm{C}-\mathrm{V}$ technique to

(a)

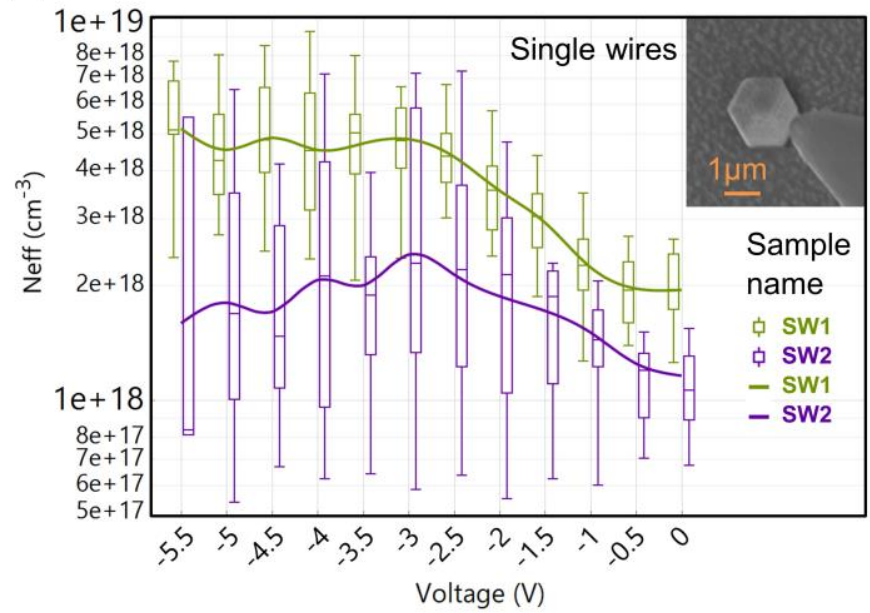

These results show the relevance of the $\mathrm{C}-\mathrm{V}$ measurements to highlight doping levels in single wire and assembly of wires with core-shell configurations and the impact of the epitaxial growth conditions on the doping levels.

(b)

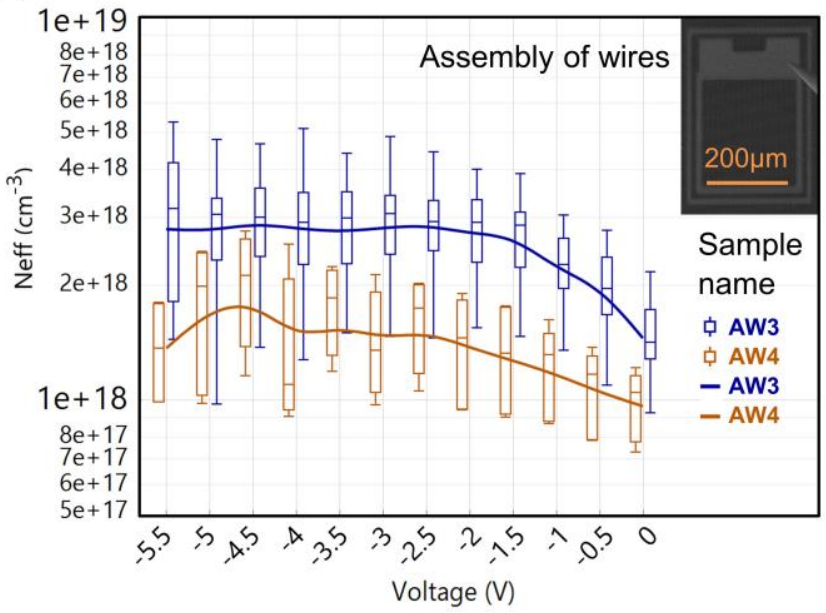

Figure 4. (a) Statistical distribution and mean values of $N_{\text {eff }}$ at different reverse voltage for 16 single wires of SW1 and 18 single wires of SW2. (b) Statistical distribution and mean values of $N_{\text {eff }}$ at different reverse voltage for 35 assemblies of wires of AW3 from $\sim 100$ to $\sim 700$ connected wires and for 5 assemblies of wires for AW4 from $\sim 100$ to $\sim 850$ connected wires. The number of wires per assembly are detailed in supporting information.

a set of four different samples in order to evaluate the impact of the epitaxial growth conditions on electrical properties. The structure and the targeted doping densities of these four samples (SW1, SW2, AW3, AW4) are reported in Table 1. Figure $4 \mathrm{a}$ and $4 \mathrm{~b}$ show $N_{\text {eff }}$ vs applied voltage for sample 1 to 4 . SW1 has a doped EBL with a target doping density set at $1 \times 10^{19} \mathrm{~cm}^{-3}$. The measured effective doping level of this sample was higher than those which were nonintentionally doped (NID) EBL (SW2, AW3, AW4). Consequently, this p-type doping increased the mean values of $N_{\text {eff }}$. According to the targeted doping densities reported in Table 1, it is possible to compare the theoretical and experimental values for the sample SW1 in the range [-5.5V;-3V] from the relation between $N_{A}, N_{D}$ and $N_{\text {eff }}$ :

$$
\frac{1}{N_{e f f}}=\frac{1}{N_{A}}+\frac{1}{N_{D}}=\frac{1}{1 \times 10^{19}}+\frac{1}{6 \times 10^{18}}=\frac{1}{3.75 \times 10^{18}}
$$

The mean experimental $N_{\text {eff }}$ measured by C-V between $-3 \mathrm{~V}$ and $-5.5 \mathrm{~V}$ is $4.9 \pm 1.6 \times 10^{18} \mathrm{~cm}^{-3}$. The theoretical and experimental values are in good agreement. A sample grown without indium was characterized to assess the impact of the quantum well on the $\mathrm{C}-\mathrm{V}$ measurements (SW2). The values and variations of $N_{\text {eff }}$ with the applied voltage (similar to AW3 sample) confirm that the QW is encompassed in the SCR at $0 \mathrm{~V}$. In order to check the Mg incorporation efficiency, the p-GaN shell of AW4 was intentionally doped at 5 times less than AW3 (Figure 4b). In both cases, the EBL and the active region are non-intentionally doped. The reduction of the doping density in the p-GaN shell of AW4 makes the mean value of $N_{\text {eff }}$ half that of AW3. In Figure $4 \mathrm{a}$ and $4 \mathrm{~b}$, the mean effective doping level increased linearly with the applied reverse voltage and reached a doping plateau for each sample. This profile reflects the transition from the NID active region to the intentionally doped $n-G a N$ and $p-G a N$.
Finally, to confirm and complete the $\mathrm{C}-\mathrm{V}$ results, EBIC measurements were performed to determine the depletion width $W$. Coupling this method with a secondary electron profile of a scanning electron microscope (SEM) directly gives the position of the SCR in the structure. EBIC measurements were carried out on 5 facets of 3 single wires from the sample SW1 after chemical mechanical polishing treatment (done after the $\mathrm{C}-\mathrm{V}$ measurement), whereby the entire active region is accessible in top view, as shown in Figure 5a (see supporting information 9). EBIC and SEM profiles separately acquired at the same location are shown Figure 5b. Due to the difference of chemical species in GaN and $\mathrm{AlGaN}$, the EBL can be delineated by secondary electron contrast. The derivative of the SEM contrast is then calculated and overlapped as shown in Figure 5b. The extrema of the derivative curve distinctly show the position and thickness of the EBL. The comparison of the EBIC signal with the derivative of the SEM contrast allowed us to evaluate the position of the SCR with respect to the active region. This corroborates results from the photocurrent about the position of QW in the SCR. Thanks to an analytical procedure ${ }^{11,39}$ the depletion width $W$ and diffusion lengths $L_{n}, L_{p}$ of minority carriers have been extracted from EBIC signal at five locations from SW1 sample, at different voltages ranging from $-5 \mathrm{~V}$ to $0 \mathrm{~V}$ (Figure $5 \mathrm{c}$ ). The diffusion lengths of the minority carriers on $n$-side and p-side region are respectively $50 \mathrm{~nm}$ and $6 \mathrm{~nm}$ to $10 \mathrm{~nm}$, inducing an asymmetry of the EBIC signal of the p-n junction. The maximum of the EBIC signal is located less than $2 \mathrm{~nm}$ to the expected position of the $\mathrm{p}-\mathrm{n}$ interface deduced from the SEM analysis. The depletion widths of 20 wires of the SW1 obtained by $\mathrm{C}-\mathrm{V}$ measurement are also reported for comparison in Figure $5 \mathrm{c}$. The variation of the depletion width with voltage follows the same trend for both EBIC and $\mathrm{C}-\mathrm{V}$ measurements and the values 
of $W$ obtained by $\mathrm{C}-\mathrm{V}$ on assembly of wires and EBIC on single wire are consistent.

Conclusion. In this study, C-V measurements were demonstrated on core-shell wires to determine the width of the depletion region and the radial dopant distribution. For the first time, $\mathrm{C}-\mathrm{V}$ measurement was carried out on individual core-shell wires which means it could be possible to study wire-to-wire homogeneity. A statistical study showed consistency between assembly of wires and single wire dopant profiling. This measurement was applied to samples with different epitaxial growth conditions to assess the impact on doping profiles from one sample to another. Effective doping levels and depletion widths were measured in the range of $1-5 \times 10^{18} \mathrm{~cm}^{-3}$ and $30-70 \mathrm{~nm}$, respectively. The depletion width values comply well with

\section{References.}

(1) Nakamura, S.; Mukai, T.; Senoh, M. Candela-Class High-Brightness InGaN/AlGaN DoubleHeterostructure Blue-Light-Emitting Diodes. Appl. Phys. Lett. 1994, 64 (13), 1687-1689. https://doi.org/10.1063/1.111832.

Wierer, J. J.; Tansu, N. III-Nitride Micro-LEDs for Efficient Emissive Displays. Laser Photon. Rev. 2019, 1900141, 1900141. https://doi.org/10.1002/lpor.201900141.

(3) Id, T. W.; Sher, C.; Lin, Y.; Lee, C.; Liang, S.; Lu, Y.; Chen, S. H.; Id, W. G.; Kuo, H.; Chen, Z. Applied (a)

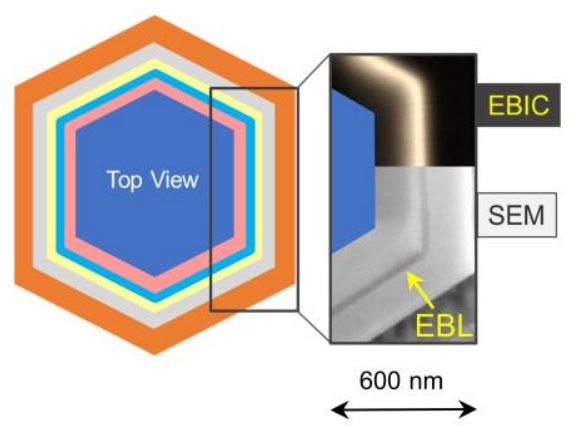

(b)

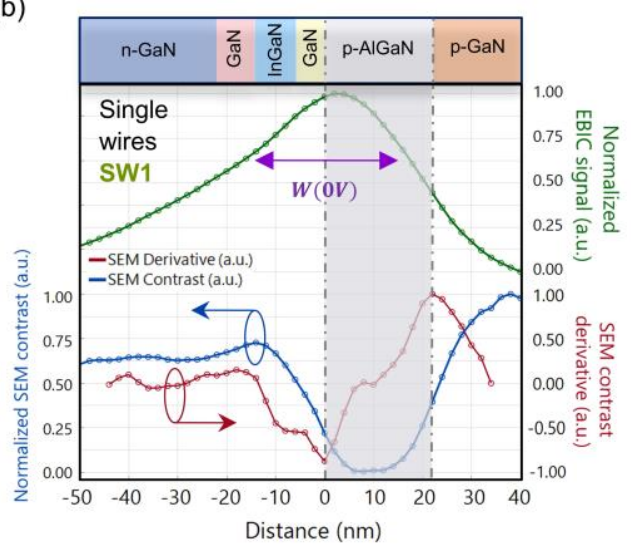

(c)

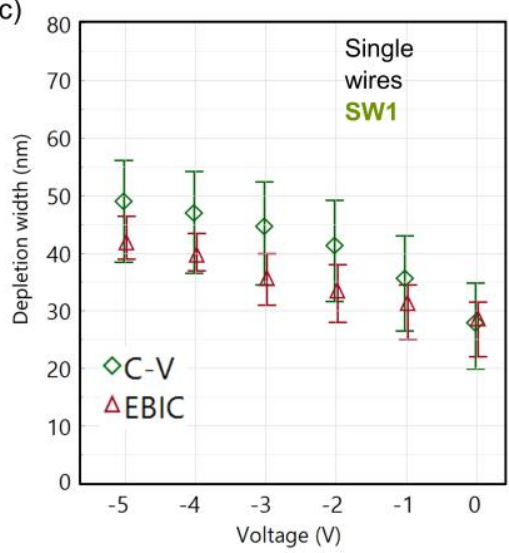

Figure 5. (a) Schematic top view of a single wire after chemical mechanical polishing treatment, localized EBIC profile and associated SEM picture at 0V. (b) Schematic cross-section of the active region from sample SW1, the EBIC signal normalized under electron beam energy of $4 \mathrm{keV}$, SEM contrast and its derivative allowing to place the EBIC signal with regards to the EBL delimited by the dotted lines, at an applied voltage of 0V. (c) Comparison of the depletion width at different voltages between $\mathrm{C}-\mathrm{V}$ and EBIC measurements of the active region from sample SW1.

EBIC measurements. Finally, the position of the SCR was established using combined EBIC and secondary electrons contrast.

\section{Supporting information.}

Additional information on growth conditions, clean room process, current-voltage characteristics, calculation of the active surface area, spatial resolution of $\mathrm{C}-\mathrm{V}$ measurement, $\mathrm{C}-\mathrm{V}$ experimental details, encompassment of the QW in the space charge region, a table that lists the number of single wires-assemblies of wires-number of connected wires per assembly and EBIC measurement details have been added in supporting information.

This material is available free of charge via the Internet at http://pubs.acs.org.

\section{Author information.}

\section{Corresponding Author}

E-mail: timothee.lassiaz@aledia.com

\section{Notes}

The authors declare no competing financial interest.
Sciences Mini-LED and Micro-LED: Promising Candidates for the Next Generation Display Technology. No. Lcd. https://doi.org/10.3390/app8091557.

Feezell, D.; Nakamura, S. Invention, Development, and Status of the Blue LightEmitting Diode, the Enabler of Solid-State Lighting. Comptes Rendus Phys. 2018, 1, 1-21. https://doi.org/10.1016/j.crhy.2017.12.001.

(5) Lin, J. Y.; Jiang, H. X. Development of MicroLED. Appl. Phys. Lett. 2020, 116 (10). https://doi.org/10.1063/1.5145201.

(6) Templier, F. GaN-Based Emissive Microdisplays: A Very Promising Technology for Compact, UltraHigh Brightness Display Systems. J. Soc. Inf. Disp. 2016, 24 (11), 669-675. https://doi.org/10.1002/jsid.516.

Olivier, F.; Tirano, S.; Dupré, L.; Aventurier, B.; Largeron, C.; Templier, F. Influence of SizeReduction on the Performances of GaN-Based Micro-LEDs for Display Application. J. Lumin. 
2017,

191 ,

$112-116$

https://doi.org/10.1016/j.jlumin.2016.09.052.

(8) Chaji, R. Low-cost micro-LED displays for all applications 2017, 264-267. ISSN 0097996X/17/4701-0264

https://doi.org/10.1002/sdtp.11683

(9) Qian, F.; Li, Y.; Gradečak, S.; Wang, D.; Barrelet, C. J.; Lieber, C. M. Gallium Nitride-Based Nanowire Radial Heterostructures for Nanophotonics. Nano Lett. 2004, 4 (10), 1975-1979. https://doi.org/10.1021/nl0487774.

(10) Shahmohammadi, M.; Ganière, J. D.; Zhang, H.; Ciechonski, R.; Vescovi, G.; Kryliouk, 0.; Tchernycheva, M.; Jacopin, G. Excitonic Diffusion in InGaN/GaN Core-Shell Nanowires. Nano Lett. 2016, $16 \quad$ (1), 243-249. https://doi.org/10.1021/acs.nanolett.5b03611.

(11) Tchoulfian, P.; Donatini, F.; Levy, F.; Dussaigne, A.; Ferret, P.; Pernot, J. [2014] Direct Imaging of $p-n$ Junction in Core-Shell GaN Wires. Direct Imaging of P-n Junction In Core-Shell GaN Wires. Nano Letters, American Chemical Society, 2014, 14 (6) https://doi.org/10.1021/nl5010493

(12) Liu, W.; Mounir, C.; Rossbach, G.; Schimpke, T.; Avramescu, A.; Lugauer, H. J.; Strassburg, M.; Schwarz, U.; Deveaud, B.; Jacopin, G. Spatially Dependent Carrier Dynamics in Single InGaN/GaN Core-Shell Microrod by TimeResolved Cathodoluminescence. Appl. Phys. Lett. 2018, $112 \quad$ (5), $1-5$. https://doi.org/10.1063/1.5009728.

(13) Tchernycheva, M.; Neplokh, V.; Zhang, H.; Lavenus, P.; Rigutti, L.; Bayle, F.; Jacopin, G.; Largeau, L.; Ciechonski, R.; Tchernycheva, M.; Neplokh, V.; Zhang, H.; Lavenus, P.; Rigutti, L. Core - Shell InGaN / GaN Nanowire Light Emitting Diodes Analyzed by Electron Beam Induced Current Microscopy and Cathodoluminescence Mapping To Cite This Version: 2016, No. 2015. https://doi.org/10.1039/C5NR00623F

(14) Lindgren, D.; Hultin, O.; Heurlin, M.; Storm, K.; Borgström, M. T.; Samuelson, L.; Gustafsson, A. Study of Carrier Concentration in Single InP Nanowires by Luminescence and Hall Measurements. Nanotechnology 2015, 26 (4), $45705 . \quad$ https://doi.org/10.1088/09574484/26/4/045705.

(15) Tchernycheva, M.; Neplokh, V.; Zhang, H.; Lavenus, P.; Rigutti, L.; Bayle, F.; Julien, F. H.; Babichev, A.; Jacopin, G.; Largeau, L.; Ciechonski, R.; Vescovi, G.; Kryliouk, O. Core-Shell InGaN/GaN Nanowire Light Emitting Diodes Analyzed by Electron Beam Induced Current Microscopy and
Cathodoluminescence Mapping. Nanoscale 2015, 7 (27), 11692-11701. https://doi.org/10.1039/c5nr00623f.

(16) Nami, M.; Stricklin, I. E.; Davico, K. M.; Mishkat-UlMasabih, S.; Rishinaramangalam, A. K.; Brueck, S. R. J.; Brener, I.; Feezell, D. F. Carrier Dynamics and Electro-Optical Characterization of HighPerformance GaN/InGaN Core-Shell Nanowire Light-Emitting Diodes. Sci. Rep. 2018, 8 (1), 1-11. https://doi.org/10.1038/s41598-017-18833-6.

Xing, Y.; Wang, L.; Yang, D.; Wang, Z.; Hao, Z.; Sun, C.; Xiong, B. A Novel Model on Time-Resolved Photoluminescence Measurements of Polar InGaN / GaN Multi- Quantum-Well Structures. Nat. Publ. Gr. 2017, No. October 2016, 1-9. https://doi.org/10.1038/srep45082.

(18) Mounir, C.; Schimpke, T.; Rossbach, G.; Avramescu, A.; Strassburg, M.; Schwarz, U. T. Polarization-Resolved Micro-Photoluminescence Investigation of InGaN/GaN Core-Shell Microrods. J. Appl. Phys. 2017, 121 (2). https://doi.org/10.1063/1.4973899.

Stiegler, J. M.; Huber, A. J.; Diedenhofen, S. L.; Gómez Rivas, J.; Algra, R. E.; Bakkers, E. P. A. M.; Hillenbrand, R. Nanoscale Free-Carrier Profiling of Individual Semiconductor Nanowires by Infrared near-Field Nanoscopy. Nano Lett. 2010, 10 (4), 1387-1392. https://doi.org/10.1021/nl100145d.

(20) Allen, J. E.; Perea, D. E.; Hemesath, E. R.; Lauhon, L. J. Nonuniform Nanowire Doping Profiles Revealed by Quantitative Scanning Photocurrent Microscopy. Adv. Mater. 2009, 21 (30), 30673072.

https://doi.org/10.1002/adma.200803865.

(21) Huang, Y.; Duan, X.; Cui, Y.; Lieber, C. M. N1015667D.Pdf. 2002, 2-5. https://doi.org/10.1021/nl015667d

(22) Tchoulfian, P.; Donatini, F.; Levy, F.; Amstatt, B.; Dussaigne, A.; Ferret, P.; Bustarret, E.; Pernot, J. Thermoelectric and Micro-Raman Measurements of Carrier Density and Mobility in Heavily Si-Doped GaN Wires. Appl. Phys. Lett. 2013, $\quad 103$ https://doi.org/10.1063/1.4829857.

(20).

Minj, A.; Cros, A.; Auzelle, T.; Pernot, J.; Daudin, B. Direct Assessment of P-n Junctions in Single GaN Nanowires by Kelvin Probe Force Microscopy. Nanotechnology 2016, 27 (38), 1-10. https://doi.org/10.1088/0957$4484 / 27 / 38 / 385202$.

(24) Storm, K.; Halvardsson, F.; Heurlin, M.; Lindgren, D.; Gustafsson, A.; Wu, P. M.; Monemar, B.; 
Samuelson, L. Spatially Resolved Hall Effect Measurement in a Single Semiconductor Nanowire. Nat. Nanotechnol. 2012, 7 (11), 718722. https://doi.org/10.1038/nnano.2012.190.

(25) Lucia, M. L.; Hernandez-Rojas, J. L.; Leon, C.; Mártil, I. Capacitance Measurements of P-n Junctions: Depletion Layer and Diffusion Capacitance Contributions. Eur. J. Phys. 1993, 14 (2), 86-89. https://doi.org/10.1088/0143$0807 / 14 / 2 / 009$.

(26) Tschirner, B. M.; Morier-Genoud, F.; Martin, D.; Reinhart, F. K. Capacitance-Voltage Profiling of Quantum Well Structures. J. Appl. Phys. 1996, 79 (9), 7005-7013. https://doi.org/10.1063/1.361466.

(27) Richter, C. A.; Hefner, A. R.; Vogel, E. M. A Comparison of Quantum-Mechanical Capacitance - Voltage Simulators. 2001, 22 (1), 35-37. https://doi.org/10.1109/55.892436

(28) Chen, N. C.; Lien, W. C.; Wang, Y. S.; Liu, H. H. Capacitance-Voltage and Current-Voltage Measurements of Nitride Light-Emitting Diodes. IEEE Trans. Electron Devices 2007, 54 (12), 3223-3228.

https://doi.org/10.1109/TED.2007.908595.

(29) Bourim, E. M.; Han, J. I. Electrical Characterization and Thermal Admittance Spectroscopy Analysis of InGaN/GaN MQW Blue LED Structure. Electron. Mater. Lett. 2015, 11 (6), 982-992. https://doi.org/10.1007/s13391-015-5180-0.

(30) Chien, F. S.-S.; Khasanah, R. A. N.; Lin, P.-T.; Lin, Y.F.; Suen, Y.-W. Impedance Elements of Significant Junctions in InGaN Light-Emitting Diodes Studied by Electric Modulus Spectroscopy. IEEE Trans. Electron Devices 2019, 66 (8), 1-6. https://doi.org/10.1109/ted.2019.2921393.

(31) Faiq, A. Extraction of Doping Profile in Substrate of MNOS Capacitor Using Fast Voltage Ramp Deep Depletion CV Method. Iraqi J. Appl. Phys. 2010, No. March 2010, 35-40. Bhuyian, M. N.; Piao, J.; Pham, T. T.; Misra, D.; Nguyen, H. P. T. Controlling Color Emission of InGaN/AlGaN Nanowire Light-Emitting Diodes Grown by Molecular Beam Epitaxy. J. Vac. Sci. Technol. B, Nanotechnol. Microelectron. Mater. Process. Meas. Phenom. 2017, 35 (2), $02 \mathrm{~B} 108$. https://doi.org/10.1116/1.4977174.

(33) Chen, X. J.; Gayral, B.; Sam-Giao, D.; Bougerol, C.; Durand, C.; Eymery, J. Catalyst-Free Growth of High-Optical Quality GaN Nanowires by MetalOrganic Vapor Phase Epitaxy. Appl. Phys. Lett. 2011, 99 (25), 242-245. https://doi.org/10.1063/1.3671365.

(34) Koester, R.; Hwang, J. S.; Durand, C.; Le Si Dang, D.; Eymery, J. Self-Assembled Growth of CatalystFree GaN Wires by Metal-Organic Vapour Phase Epitaxy. Nanotechnology 2010, 21 (1). https://doi.org/10.1088/09574484/21/1/015602.

Birner, S.; Zibold, T.; Andlauer, T.; Kubis, T.; Sabathil, M.; Trellakis, A.; Vogl, P. Nextnano: General Purpose 3-D Simulations. IEEE Trans. Electron Devices 2007, 54 (9), 2137-2142. https://doi.org/10.1109/TED.2007.902871.

(36) Inoguchi, T. Semiconductor Physics and Semiconductor Devices; 1974; Vol. 28. https://doi.org/10.3169/itej1954.28.723.

(37) Liu, Y.-J.; Tsai, T.-Y.; Yen, C.-H.; Chen, L.-Y.; Tsai, T.-H.; Huang, C.-C.; Chen, T.-Y.; Hsu, C.-H.; Liu, W.C. Performance Investigation of GaN-Based LightEmitting Diodes with Tiny Misorientation of Sapphire Substrates. Opt. Express 2010, 18 (3), 2729-2742. https://doi.org/10.1364/OE.18.002729.

(38) Zhao, J. Z.; Lin, Z. J.; Corrigan, T. D.; Zhang, Y.; Lü, Y. J.; Lu, W.; Wang, Z. G.; Chen, H. Determination of the Relative Permittivity of the AlGaN Barrier Layer in Strained AlGaN/GaN Heterostructures. Chinese Phys. B 2009, 18 (9), 3980-3984. https://doi.org/10.1088/1674-1056/18/9/060.

(39) Bonard, J. M.; Ganière, J. D. Quantitative Analysis of Electronbeaminduced Current Profiles across $\mathrm{p}-\mathrm{n}$ Junctions in GaAs/Al0.4Ga0.6As Heterostructures. J. Appl. Phys. 1996, 79, 6987. https://doi.org/10.1063/1.361464. 
